\title{
WREATH PRODUCTS AND REPRESENTATIONS OF DEGREE ONE OR TWO $\left.{ }^{1}\right)$
}

\author{
B Y
}

\author{
J. M. BATEMAN, RICHARD E. PHILLIPS AND L. M. SONNEBORN
}

ABSTRACT. $\mathcal{S}_{2}$ denotes all groups $G$ that possess an ascending invariant series whose factors are one- or two-generated Abelian groups. We are interested in the problem (1): For which nontrivial groups $A$ and $B$ is $A$ wr $B$ in $\mathcal{\delta}_{2}$ ? (1) has been completely solved by D. Parker in the case where $A$ and $B$ are finite of odd order. Parker's results are partially extended here to cover groups of even order. Our answer to (1) is complete in the case where $A$ is a finite 2 group: If $A$ is a finite 2-group, $A$ wr $B$ is in $\mathcal{S}_{2}$ iff $B$ is finite and $B / O_{2}(B)$ is isomorphic to a subgroup of a dihedral group of an elementary 3-group. If $A$ is not a 2-group, we offer only necessary conditions on $B$. Problem (1) is closely related to Problem (2): If $F$ is a prime field or the integers, which finite groups $B$ have all their irreducible representations over $F$ of degrees one or two? It is shown that all finite $B$ which satisfy (2) are $\delta_{2}$ groups; in particular all such $B$ are solvable.

I. Introduction and notation. Let $\mathcal{S}_{2}$ denote the class of all groups which have an ascending (possibly infinite) invariant series whose factors are all oneor two-generated Abelian groups. In this paper we consider two related problems:

Problem 1. For what (nontrivial) groups $A$ and $B$ is the (standard, restricted) wreath product $A$ wr $B$ in $\mathcal{S}_{2}$ ?

Problem 2. For a given prime field (resp. the integers), which finite groups $B$ have all their irreducible representations over it of degree one or two?

We will use Suprenenko's [10] characterization of the solvable subgroups of $\mathrm{GL}(2, p)$ to establish that all $B$ which arise in Problem 2 are themselves in $\mathcal{S}_{2}$, which will mean that such groups will also answer Problem 1 for selected $A$. Moreover a reduction in Problem 1 will allow the consideration of the special cases $A=Z_{p}$ or $Z$ with $B$ finite so that information about Problem 1 will be applicable to Problem 2.

Received by the editors February 14, 1972.

AMS (MOS) subject classifications (1970). Primary 20E15, 20F25; Secondary 20C10, $20 \mathrm{C} 20$.

Key words and phrases. Supersolvable, solvable wreath product, group ring, modular representation, integral representation.

(1) All correspondence concerning this paper should be addressed to the third author. 
The class $\mathfrak{S}_{2}$ is a natural generalization of supersolvable groups, for which Problem 1 was answered in the finite case by Durbin [4] and in the infinite case by Scott and Sonneborn [9]. D. Parker [8] has answered Problem 1 for finite groups of odd order using the fact that odd order groups in $\mathcal{S}_{2}$ form a saturated formation [6, p. 712]. The finite groups in $\mathcal{S}_{2}$ are also a formation, since $\mathcal{S}_{2}$ is obviously closed under the taking of subgroups, homomorphic images, and direct sums, but this formation is not saturated, [6, pp. 713-714], so that Parker's methods do not apply.

We will call a series which demonstrates that a group $G \in \mathcal{S}_{2}$ an $\mathcal{S}_{2}$-series for $G$. In $A$ wr $B$, the base group $A^{B}$ will be $K$ and $A_{1}=\{f \in K \mid f(b)=1$ for all $b \neq 1\}$ will be identified as $A$.

II. Reduction of the problem. We restate Parker's result (both specialized and corrected) as it applies to Problem 1.

Theorem 1. If $A, B$ are nontrivial finite groups of odd order, then $A$ wr $B \epsilon$ $\mathcal{S}_{2}$ if, and only if,

(i) $A$ is nilpotent, $B \in \mathcal{S}_{2}$, and

(ii) for all primes $p|| A \mid, B$ bas a normal Sylow p-subgroup $P$ with $B / P$ Abelian of exponent dividing $p^{2}-1$.

Lemma 1. If $1 \neq C \triangleleft A$ and $C \cap Z(A)=1$ and $1 \neq L \triangleleft A$ wr $B$ with $L \subset C^{B}$, then there exists $D \triangleleft A, D \neq 1$ such that $D^{3} \subset L$.

Proof. Let $1 \neq b \in L$ and $b \in B$ be in the support of $b$. Since $b_{b} \notin Z(A), \exists r \in$ $A^{b}$ such that $1 \neq[r, b] \in L \cap A^{b}$. Thus $L \cap A^{b} \neq 1$ and $\left(L \cap A^{b}\right)^{B}=(L \cap A)^{E}=$ $D^{B} \subset L$.

Lemma 2. If $A$ and $B$ are nontrivial, then $A \mathrm{wr} B \in \mathcal{S}_{2}$ if, and only if, either

(i) $A$ bas an ascending invariant series with cyclic factors (an $S S^{*}$ series) and $|B|=2$, or

(ii) $A$ is a ZA-group, $B \in \mathcal{S}_{2},|B|$ is finite and either

(a) $A$ is a periodic group and $Z_{p}$ wr $\left(B / O_{p}(B)\right) \in \mathcal{S}_{2}$ for all primes relevant to $A$, or

(b) $A$ bas an element of infinite order and $Z$ wr $B \in \mathcal{S}_{2}$.

Proof. Suppose $A$ wr $B \in \mathcal{S}_{2}$. Then $A \in \mathcal{S}_{2}$ and $B \in \mathcal{S}_{2}$.

(1) $A$ is an $S S^{*}$ group. If not, then there is an $\mathcal{S}_{2}$-series $1=A_{0} \triangleleft A_{1} \triangleleft \cdots$ $\triangleleft A_{a}=A$ which admits no (ascending) invariant refinement with cyclic factors. We may assume that, for some $\beta, A_{\beta+1} / A_{\beta} \approx Z_{p}+Z_{p}$ or $Z+Z$ and that $A_{\beta+1} / A_{\beta}$ contains no normal cyclic subgroup of $A / A_{\beta}$. Then in $(A \operatorname{wr} B) /\left(A_{\beta}\right)^{B} \approx$ $\left(A / A_{\beta}\right)$ wr $B$ the normal subgroup $\left(A_{\beta+1}\right)^{B} /\left(A_{\beta}\right)^{B}$ has normal subgroups (by Lemma 1) only of type $D^{B} /\left(A_{\beta}\right)^{B}$ where $A_{\beta}<D \triangleleft A$. Since $D / A_{\beta}$ is two-generated 
$D^{B} /\left(A_{\beta}\right)^{B}$ is $2|B|$-generated and there is no $\mathcal{S}_{2}$-series refinement of $1=A_{0}^{B} \triangleleft \cdots$ $A_{\alpha}^{B} \triangleleft A$ wr $B$, contrary to assumption.

(2) If $|B|>2$, then $A$ is a $Z A$ group. If $A$ has an $S S^{*}$-series with cyclic factors which does not have a hypercentral refinement, a similar application of Lemma 1 shows that $D^{B} / A_{\beta}^{B}$ is $|B|$-generated, for some $\beta$, and that $|B|$ must therefore be two.

(3) $|B|$ is finite. Otherwise, any normal subgroup of $K=A^{B}$ would contain elements the union of whose supports is infinite, and therefore could not be $\mathrm{f}$ nitely generated.

In case (i), if $A$ has an element of order $p$ (resp. of infinite order), then $Z_{p}$ wr $B(Z$ wr $B) \subseteq A$ wr $B$ so that $Z_{p}$ wr $B$ (resp. $Z$ wr $\left.B\right) \in \mathcal{S}_{2}$. In the former case, $Z_{p}$ wr $\left(B / O_{p}(B)\right)$ is a homomorphic image of $Z_{p}$ wr $B$ (see Gruenberg [5, Lemma 3.2]) and it, too, is in $\mathcal{S}_{2}$.

Now suppose $A, B$ satisfy (i). Then $1=A_{0}+A_{0}^{b}<A_{1}+A_{1}^{b} \ldots \triangleleft A_{a}+A_{a}^{b} \triangleleft$ $A$ wr $B$ is an $\mathcal{S}_{2}$-series where $B=\{1, b\}$ and the $A_{\beta}$ form an $S S^{*}$-series for $A$. If $A, B$ satisfy (ii), and $\left\{A_{\beta} \mid \beta \leq \alpha\right\}$ is an ascending hypercentral series for $A$ with cyclic factors and, for each factor $\left(A_{\beta+1} / A_{\beta}\right)$ wr $B \in \mathcal{S}_{2}$, then the series $1=A_{0}^{B} \triangleleft$ $A_{1}^{B} \triangleleft \ldots \triangleleft A$ wr $B$ may be refined to an $\mathcal{S}_{2}$-series using the fact that $\left(A_{\beta+1}\right)^{B} /\left(A_{\beta}\right)^{B} C\left(Z\left(A / A_{\beta}\right)\right)^{B}$ so that normalization by $B$ is equivalent to normalization by $\left(A / A_{\beta}\right)$ wr $B$ in $\left(A / A_{\beta}\right)$ wr $B \simeq(A$ wr $B) /\left(A_{\beta}\right)^{B}$.

The proof will now be complete if it is shown that (ii) implies that $A_{\beta+1} / A_{\beta}$ wr $B \in \mathcal{S}_{2}$ for all $\beta$. Without loss of generality, $A_{\beta+1} / A_{\beta} \approx Z$ or $Z_{p}$ for some prime $p$. If, for some $\gamma, A_{\gamma+1} / A_{\gamma} \approx Z$ then, by (b), $Z$ wr $B \in \mathcal{S}_{2}$ and for all primes $p$, $Z_{p}$ wr $B=(Z / p Z)$ wr $B=(Z$ wr $B) /(p Z)^{B} \in \mathcal{S}_{2}$ so that $\left(A_{\beta+1} / A_{\beta}\right)$ wr $B \in \mathcal{S}_{2}$ for all $\beta$. If $A_{\beta+1} / A_{\beta}$ is always finite, then for each prime $p$ which arises, we have $Z_{p}$ wr $\left(B / O_{p}(B)\right) \in \mathcal{S}_{2}$. But $Z_{p}$ wr $B$ is an extension of the finite $p$-group $\left(Z_{p}\right)^{B} \cdot O_{p}(B)$ by $B / O_{p}(B)$ and is therefore, by the Kaloujnine-Krasner embedding theorem (see [7, Theorem 22.21]) isomorphic to a subgroup of $\left(\left(Z_{p}\right)^{B} \cdot O_{p}(B)\right) \mathrm{wr}\left(B / O_{p}(B)\right)$, where the first group has a central series with all factors isomorphic to $Z_{p}$. This last wreath product is in $\mathcal{S}_{2}$ by the argument of the previous paragraph and so also is its subgroup $Z_{p}$ wr $B$.

We are now in a better position to explore the connection between Problems 1 and 2. Lemma 2 allows us to explore only groups of the types $Z_{p}$ wr $B$ in which $O_{p}(B)=1$ and $Z$ wr $B$ for finite groups $B$. In both cases, the base group is isomorphic (as a $B$-module) to the group ring $Z_{p}(B)$ (resp. $Z(B)$ ) in such a way that right ideals of the group ring are precisely the subgroups of the base group which are normal in $Z_{p}$ wr $B$ (resp. $Z$ wr $B$ ), so that in one of these cases $A$ wr $B \in \mathcal{S}_{2}$ if, and only if, $B \in \mathcal{S}_{2}$ and the group ring $A(B)$ has an ascending sequence of right ideals with quotients of generation "rank" one or two, and hence, if, and only 
if, $B \in \mathcal{S}_{2}$ and all the irreducible representations of $B$ over $A$ are of degree one or two. (For $A=Z$, use [3, pp. 497-498]. Given the above ascending sequence of right ideals and a $Z$-irreducible representation module of $Z$-rank $r$, we find a cyclic quotient $Z(B) / N$ of rank $r$, refine the sequence $O \subseteq N \subset Z(B)$, and obtain $N \subset$ $N^{\prime} \subseteq Z(B)$ with $N^{\prime} / N Z$-free of rank $r$ and also of "rank" one or two, so that $r \leq$ 2. Conversely, if the $Z$-irreducible representation degrees are one or two, we form a $Z$-composition series for $Z(B)$ with factor ranks one or two and this is an $\mathcal{S}_{2}$ series.) Moreover factoring out $O_{p}(B)$ is irrelevant in Problem 2 since $O_{p}(B)$ is in the kernel of all irreducible representations of $B$ over $Z_{p}$. When the remark following the statement of Problem 2 is established below (Theorem 5) we will have shown

Theorem 2. $Z_{p}$ wr $B \in \dot{\mathcal{S}}_{2}$ (resp. $Z$ wr $B \in \mathcal{S}_{2}$ ) if, and only if, $B$ is finite and all irreducible representations of $B$ over $Z_{p}$ (resp. $\left.Z\right)$ are of degree one or two.

III. Information on Problem 2 in the case of $Z_{p}$ representations. Throughout this section a group $B$ will be called "good" if all its irreducible representations over $Z_{p}$ are of degree one or two. Subgroups and homomorphic images of good groups are good (consider the group ring version). We assume that $B$ is finite solvable and $O_{p}(B)=1$.

Lemma 3. Let $p \neq 2$ be a prime.

(i) An Abelian ( $\left.p^{\prime}\right)$ group $B$ is good if, and only if, its exponent divides $p^{2}-1$.

(ii) If $B$ is a split extension of a cyclic $p^{\prime}$-group by a group of order 2 and $B \subsetneq \mathrm{GL}(2, p)$, then $B$ is good.

(iii) If $B$ bas an Abelian normal $p^{\prime}$-subgroup of exponent dividing $p-1$ and of index 2 , then $B$ is good.

(iv) If $B=B_{1}+\cdots+B_{n}$ and each $B_{i}$ is good, then $B$ is good if, and only if, either

(a) each $B_{i}$ is Abelian with exponent dividing $p^{2}-1$, or

(b) exactly one $B_{i}$ is non-Abelian and the others are Abelian of exponent dividing $p-1$.

Proof. (i) If $B$ has an element $x$ of order not dividing $p^{2}-1$, then the subgroup $\langle x\rangle$ has a faithful absolutely irreducible representation in GF $\left(p^{n}\right)$ for some $n>2$. Since $\exp (\mathrm{GL}(2, p)) \mid p \cdot\left(p^{2}-1\right)$ there is no such representation in it by a $p^{\prime}$-element, and $B$ is not good. Conversely if $\exp B \mid p^{2}-1$, all its absolutely irreducible representations are of degree one over $\mathrm{GF}\left(p^{2}\right)$ and therefore its irreducible representations are of degree one or two over $Z_{p}$.

(ii) We use the description of representations of metacyclic groups given in Curtis and Reiner [3, pp. 333-340]. In conformity with the notation used there, 
the group $B=\left\langle a, b \mid a^{m}=b^{2}=1, b^{-1} a b=a^{r}\right\rangle$ has all its absolutely irreducible representations of degree one or two (Theorem 47.11 ) and they are realizable over a field with $m$ th roots of unity. In the case at hand, since $m \mid p^{2}-1$ by the argument of part (i), GF $\left(p^{2}\right)$ will suffice. The two-dimensional representations are given by

$$
a \rightarrow\left(\begin{array}{cc}
\xi & 0 \\
0 & \xi
\end{array}\right)^{i}, \quad b \rightarrow\left(\begin{array}{ll}
0 & 1 \\
1 & 0
\end{array}\right)
$$

where $\xi$ is a primitive $m$ th root of unity and where the irreducible representations have $r i \not \equiv i(\bmod m)$ or, equivalently, $i(r-1) \not \equiv 0(\bmod m)$. If $r \equiv 1(\bmod m), B$ is the Abelian group $Z_{m} \times Z_{2}$ which is good by part (i); while if $r \not \equiv 1(\bmod m)$, we see that the representation above is faithful if and only if $(i, m)=1$. Therefore if $r \not \equiv 1(\bmod m)$, any irreducible such representation is a power (so far as " $a$ " is concerned) of any of the faithful irreducible ones. It follows now, since $B$ is nonAbelian $(r \not \equiv 1(\bmod m))$ and $B \subseteq \mathrm{GL}(2, p)$, that there is a matrix $U$ such that $U^{-1} T(a) U$ and $U^{-1} T(b) U$ have coefficients in $Z_{p}$. But so then does $U^{-1} T(a)^{j} U=$ $\left(U^{-1} T(a) U\right)^{j}$, whereby all the above representations are realizable over $Z_{p}$. The one-dimensional absolutely irreducible representations of $B$ are those of $B / B^{\prime} \subseteq Z_{p^{2-1}} \times Z_{2}$ which are realizable as representations of degree one or two over $Z_{p}$ by part (i).

(iii) By Durbin's [4] characterization of supersolvable wreath products, $Z_{p}$ wr $H$ is supersolvable, where $H$ is the normal Abelian subgroup of $B$ of exponent dividing $p-1$, and $Z_{p}$ wr $B$ has a subgroup of index 2 which is isomorphic to a subgroup of $\left(Z_{p}\right.$ wr $\left.H\right) \times\left(Z_{p}\right.$ wr $\left.H\right)$. By Lemma $2(i), Z_{p}$ wr $B \in \mathcal{S}_{2}$ and by the remarks preceding Theorem $2, B$ is good.

(iv) $Z_{p}(B)=\otimes Z_{p}\left(B_{i}\right)$ and $Z_{p}\left(B_{i}\right)=\operatorname{Rad}\left(Z_{p}\left(B_{i}\right)\right)+S_{i}$, where $S_{i}$ is a direct sum of algebras which, since $B_{i}$ is good, can only be $Z_{p}, \mathrm{GF}\left(p^{2}\right)$ or $M_{2}\left(Z_{p}\right)$. Using the facts that $Z_{p} \otimes A \approx A, \mathrm{GF}\left(p^{2}\right) \otimes \mathrm{GF}\left(p^{2}\right) \approx \mathrm{GF}\left(p^{2}\right)+\mathrm{GF}\left(p^{2}\right)$, $G F\left(p^{2}\right) \otimes M_{2}\left(Z_{p}\right) \approx M_{2}\left(G F\left(p^{2}\right)\right)$ and $M_{2}\left(Z_{p}\right) \otimes M_{2}\left(Z_{p}\right) \approx n_{4}\left(Z_{p}\right)$ and induction, we see that $Z_{p}(B)=\operatorname{Rad}\left(Z_{p}(B)\right)+S$ has all its (right) composition factors of dimension one or two if and only if $\operatorname{GF}\left(p^{2}\right) \otimes M_{2}\left(Z_{p}\right)$ and $M_{2}\left(Z_{p}\right) \otimes M_{2}\left(Z_{p}\right)$ do not occur (i.e., if some $B_{i}$ is non-Abelian so that $M_{2}\left(Z_{p}\right)$ occurs, all other $B_{i}$ must be Abelian (to prevent the second from occurring) and of exponent dividing $p-1$ ). Conversely, if all are Abelian of exponent dividing $p^{2}-1$, part (i) yields that $B$ is good, while if exactly one is non-Abelian and all others have exponent dividing $p-1$, the algebra is of the form $\operatorname{Rad}\left(Z_{p}(B)\right)+\Sigma\left(Z_{p} \otimes Z_{p}\left(B_{i}\right)\right)$ and $B$ is again good.

Continuing with the assumption that $B$ is solvable and $O_{p}(B)=1$, we have that the intersection of the kernels of the irreducible representations is 1 , so that 
if $B$ is good, $B \subseteq R_{1}+R_{2}+\cdots+R_{n}$ where each $R_{i}$ is an irreducible solvable subgroup of $\mathrm{GL}(1, p)$ or $\mathrm{GL}(2, p)$ and hence $O_{p}\left(R_{i}\right)=1$. The candidates for such $R_{i} \subseteq \mathrm{GL}(2, p)$ are given by

Theorem 3 (Suprenenko [10, pp. 39-44]). Every maximal irreducible solvable subgroup of $\mathrm{GL}(2, p)$ is among

(i) $H_{1} \approx Z_{p-1}$ wr $Z_{2}$ for $p>2$,

(ii) $\mathrm{H}_{2}$ which is a split extension of a cyclic group of order $p^{2}-1$ by a group of order 2,

(iii) $H_{3}$ which is of order $24(p-1)$ and is a central extension of a cyclic group of order $p-1$ by $\operatorname{Sym}(4)(p>2)$.

Theorem 4. The groups listed in Theorem 3, and the ir subgroups, are all good except those subgroups of $\mathrm{H}_{3}$ which involve Alt (4).

Proof. (i) $H_{1}$ is good by Lemma 3 (iii). Its subgroups are therefore good.

(ii) $\mathrm{H}_{2}$ is good by Lemma 3 (ii) for $p>2$. For $p=2, H_{2} \approx$ Sym 3 which is good (over $Z_{2}$ ) since $Z_{2}$ (Sym 3 ) has a chain of right ideals with all quotients of dimension one or two.

(iii) The maximal subgroups of $H_{3}$ which do not involve Alt (4) are of orders $8(p-1)$ and $6(p-1)$ corresponding to the extension of the central subgroup of order $p-1$ first by a Sylow 2-subgroup of Sym (4) and then by Sym (3). The generators given by Suprenenko in the first case yield that $B=\left(Z_{p-1}\right)_{\text {odd }}+S$ with $S \in \operatorname{Syl}_{2}(B)$. The Sylow 2-subgroups of $\mathrm{GL}(2, p)$ have been determined by Carter and Fong [2] and are $Z_{2}$ wr $Z_{2}$, where $2^{s}$ is the largest power of 2 dividing $p-1$ if $p \equiv 1(\bmod 4)$, and $\left\langle a, b \mid a^{2^{s+1}}=b^{2}=1, b^{-1} a b=a^{2^{s}-1}\right\rangle$ where $2^{s}$ is the largest power of 2 dividing $p+1$ if $p \equiv 3(\bmod 4)$. The first group is good by Lemma 3 (iii) and the second is good by Lemma 3 (ii) so that the group $S$ is good. But then Lemma 3 (iv) implies that $B$ is good.

Now suppose $|B|=6(p-1)$. (This case does not arise if $p=3$ for then $O_{3}(B) \neq 1$. The allowable subgroups of order dividing 4 have been considered above.) $B$ has a normal Abelian subgroup $H$ of order $3(p-1)$. If $3 \nmid(p-1), H$ is primitive, so that by Suprenenko [10, Lemma 8$], B$ is contained in a solvable subgroup $C$ of $\mathrm{GL}(2, p)$ whose maximal normal Abelian subgroup contains $H$ and is the multiplicative group of a field. This group $\mathrm{C}$ must be $\mathrm{H}_{2}$, as $\mathrm{H}$ itself is a maximal Abelian subgroup of $H_{3}$. But $H_{2}$ is good, so $B$ is also. If $3 \mid p-1$, then $H$ cannot be cyclic because the power of 3 occurring in $H$ is larger than that in $p^{2}-1$, the maximal order of a $p^{\prime}$-element in $\mathrm{GL}(2, p)$, so that in this case $H$ is an Abelian normal subgroup of $B$ of exponent $p-1$ and of index 2 . $B$ is good by Lemma 3 (iii).

We complete the fproof by showing that, for $p>2$, Alt (4) is not good. (This 
suffices in view of the closure properties of "goodness".) If $p \neq 3$, then $(p, \mid$ Alt $(4) \mid)=1$ and the absolutely irreducible representations have degrees 1,1 , 1,3 as the sum of squares formula requires. Obviously some representation over $Z_{p}$ has degree no less than three. If $p=3$, Alt $(4)$ is not contained in any subdirect sum of subgroups of $\mathrm{GL}(2,3)$, because if it were, it would be in one of them, which it is not. (If Alt $(4) \subseteq G L(2,3)$, then Alt (4) is a complement to $Z(G L(2,3)$ ) in $\operatorname{SL}(2,3)$ which is impossible.) Therefore by the remark preceding Theorem 3, Alt (4) is not good for $p=3$.

Corollary. A solvable subgroup $B$ of $\mathrm{GL}(2, p)$ with $O_{p}(B)=1$ and all its irreducible representations over $Z_{p}$ of degree one or two is supersolvable.

We now remove the hypotheses that $B$ is solvable and $O_{p}(B)=1$, but still assume that $B$ is finite.

Theorem 5. A group $B$ which has all its irreducible representations over $Z_{p}$ of degree one or two is solvable. (In fact $B / O_{p}(B)$ is supersolvable and $B \in \varsigma_{2}$. .)

Proof. Let $B$ be a counterexample of smallest order. Since $B / O_{p}(B)$ has the same irreducible representations and is nonsolvable, $O_{p}(B)=1$. As in the remark preceding Theorem 3, $B$ is a subdirect sum of subgroups of $\mathrm{GL}(2, p)$ at least one of which is then nonsolvable. As a homomorphic image of $B$ it, too, is good and is, therefore, is omorphic to $B$. The highest power of $p$ dividing the order of $B$ is now $p$ itself. Let $M$ be a maximal subgroup of $B$. Then $M / O_{p}(M)$ is solvable and has all its irreducible representations over $Z_{p}$ of degree one or two, and, by the Corollary, is a subdirect sum of supersolvable groups and is, the refore, itself supersolvable. Since $\left|O_{p}(M)\right|=1$ or $p, M$ is supersolvable, and therefore $B$ has all its maximal subgroups supersolvable so that $B$ (see $[6, \mathrm{p} .718]$ ) is solvable, a contradiction. $B / O_{p}(B)$ is supersolvable exactly as $M / O_{p}(M)$ was above. $Z_{p}$ wr $B / O_{p}(B) \in S_{2}$ since the $S_{2}$-series up to the group ring which is the base group can be continued using the $S S^{*}$ series for $B / O_{p}(B)$. By Lemma $2\left(\right.$ ii),$Z_{p}$ wr $B \in \mathcal{S}_{2}$, and, therefore, $B \in \AA_{2}$.

Theorem 6. (i) If $\mathrm{O}_{2}(B)=1$, then $Z_{2}$ wr $B \in \mathcal{S}_{2}$ (or, equivalently, all the irreducible representations of $B$ over $Z_{2}$ are of degree one or two) if, and only if, $B$ is isomorpbic to a subgroup (other than $Z_{2}$, of course) of the dibedral group of an elementary Abelian three group.

(ii) If $O_{3}(B)=1$ and $Z_{3}$ wr $B \in \mathcal{\delta}_{2}$, then $B$ is a subdirect sum of subgroups of $\mathrm{Syl}_{2}(\mathrm{GL}(2,3))$, the semidibedral group or order 16 . In particular $B$ is a 2 group.

(iii) If $p>3$ and $O_{p}(B)=1$ and $Z_{p}$ wr $B \in \mathcal{S}_{2}$, then $B$ is a subdirect sum of irreducible supersolvable subgroups of $\mathrm{GL}(2, p)$, as listed in Theorem 4 , and subgroups of GL $(1, p)$. 
Proof. (i) If $B$ is the dihedral group of an elementary Abelian 3-group, then $Z_{2}$ wr $B \in \mathcal{S}_{2}$ because $Z$ wr $B \in \mathcal{S}_{2}$. (See (5) of $\$ I V$ below. Its proof is independent of this section.) Conversely, if $Z_{2}$ wr $B \in \mathcal{S}_{2}$, then $B \subseteq$ Sym (3) $+\cdots+\operatorname{Sym}(3)$ (Sym $3=\mathrm{GL}(2,3))$. If $B$ is a subdirect sum, then it is pairwise nondirect by Lemma 3 (iv) and therefore, by Bateman [1, Theorem 9], is the dihedral group of some elementary Abelian 3-group. If $B$ is not a subdirect sum of Sym (3)'s it is a subdirect sum of Sym (3)'s and $Z_{3}$ 's (since $O_{2}(B)=1$ ). Assuming this has been done in such a way as to use as few $Z_{3}$ 's as possible, we see that either there must be no $Z_{3}$ 's or no Sym (3)'s since (Sym 3) $+Z_{3}$ is bad by Lemma 3 (iv). Thus $B$ is either a subdirect sum of Sym (3)'s or a subgroup of such, namely an elementary Abelian 3-group.

(ii) All facets of this assertion are in Theorem 4 and its proof.

(iii) This also is contained in Theorem 4.

By Theorem 6(i), we have necessary and sufficient conditions for $Z_{2}$ wr $B$ to be in the class $\mathcal{S}_{2}$. If $p \neq 2$, the situation is much more complicated; if $Z_{p} \mathrm{wr}$ $B \in \mathcal{S}_{2}$, then $B$ is a subdirect product of subgroups of the groups listed in Theorem 4 . The determination of which subdirect products are good appears to be very difficult and is left unresolved. Some of the difficulties are illustrated in the first example.

Example 1. In the case $p=2$ above, the result states that any subdirect sum of Sym 3's which is pairwise not direct is good. This is not the case for $p=3$. Let $G=\left\langle a, b \mid a^{8}=b^{2}=1, b^{-1} a b=a^{3}\right\rangle$ be a presentation of the semidihedral group, and $H \subseteq G+G$ be given by $H=\langle(a, b),(b, a)\rangle .|H|=64$ and $H$ is not good.

Example 2. The dihedral group of order 8 is a subgroup of the semidihedral groups and of $Z_{2}$ wr $Z_{2}$ for all $s \geq 2$ so that it is good with respect to all primes (see also (6) below). The quaternion group of order 8 also has this property, as does Sym (3).

IV. Problem 2 for $Z$-representations. We now consider the question "For what $B$ is $Z$ wr $B \in \mathcal{S}_{2}$ ?" (or "For what $B$ are all irreducible integral representations of degree one or two?"'). From the proof of Lemma 2, we see that a necessary condition is that $Z_{p}$ wr $B \in \mathcal{S}_{2}$ for all primes. In the $S S^{*}$ case (see [9]), the analogous condition was also sufficient, but here it is not.

(1) If $Z$ wr $B \in \mathfrak{S}_{2}$, then $B$ has elements of orders 1, 2, 3, 4, 6 only. Also $Z$ wr $Z_{n} \in \mathcal{S}_{2}$ for $n=1,2,3,4,6$.

Proof. Since $Z_{2}$ wr $B \in \delta_{2}, B$ is a 2, 3-group. The order of any element must divide $5^{2}-1$ since $Z_{5}$ wr $B \in \mathcal{S}_{2}$. Thus we need exclude only 8 and 12 . $Z$ wr $Z_{8} \notin$ $\mathcal{S}_{2}$ because, while $x^{8}-1$ factors into linear and quadratic factors over $Z_{p}$ for all $p$, it 
does not do so over the rationals, so that $Z_{8}$ has an irreducible representation of degree 4 over the rationals and therefore over the integers. $Z$ wr $Z_{12} \notin \mathcal{S}_{2}$ for an analogous reason. It is routine to produce ascending chains of right ideals in $Z\left(Z_{4}\right)$ and $Z\left(Z_{6}\right)$ whose quotients are of rank one or two, so $Z$ wr $Z_{4}$ and $Z$ wr $Z_{6}$ are in $\mathcal{S}_{2}$. Thus the orders indicated are seen to be permissible.

(2) If $Z$ wr $B \in \mathcal{S}_{2}$, then $Z$ wr $\left(B+Z_{2}\right) \in \mathcal{S}_{2}$.

Proof. Let $1=A_{0} \triangleleft A_{1} \cdots \triangleleft A_{m}=K$ be a $(B)$-invariant $\mathcal{S}_{2}$-series in $Z$ wr $B$ up to its base group $K$, and let $1 \neq g \in Z_{2}$. We see that the base group $L$ in $Z \mathrm{wr}\left(B+Z_{2}\right)$ is given by $K+K^{g}$ and that $\left(A_{i}+A_{i}^{g}\right)$ is invariant in $Z$ wr $\left(B+Z_{2}\right)$. We will show that the series $1=A_{0}+A_{0}^{g} \triangleleft \cdots \triangleleft A_{m}+A_{m}^{g}=L$ up to the base group has an $S_{2}$-refinement, noting that it is routine to extend the resulting series to an $\mathcal{S}_{2}$-series ending at $Z$ wr $\left(B+Z_{2}\right)$.

Let $B_{i}=\left(A_{i}+A_{i}^{g}\right) \cdot\left\{x+x^{g} \mid x \in A_{i+1}\right\}$. Since $\left(x+x^{g}\right)^{b}=x^{b}+x^{g b}=x^{b}+$ $x^{b g}=x^{b}+\left(x^{b}\right)^{g}$ and $\left(x+x^{g}\right)^{g}=x^{g}+x=x+x^{g}$, the group $B_{i}$ is invariant and $B_{i} / A_{i}+A_{i}^{g}$ has no more generators than $A_{i+1} / A_{i}$. Next let $C_{i}=B_{i} \cdot\left\{x-x^{g} \mid x \in\right.$ $A_{i+1}$ \}. Clearly $C_{i}$ is also invariant and $C_{i} / B_{i}$ has no more generators than $A_{i+1} / A_{i}$. We now have the series $1=A_{0}+A_{0}^{g} \triangleleft B_{0} \triangleleft C_{0} \triangleleft A_{1}+A_{1}^{g} \triangleleft B_{1} \triangleleft C_{1} \triangleleft$ $A_{2}+A_{2}^{g} \ldots$ in which the series is known to be an $\delta_{2}$-series except possibly at the jump $\left(A_{i+1}+A_{i+1}^{g}\right) / C_{i}$. If $x+y^{8} \in A_{i+1}+A_{i+1}^{g}$, then $\left(x+y+(x+y)^{g}\right)+$ $\left((x-y)-(x-y)^{g}\right)=2 x+2\left(y^{g}\right) \in C_{i}$ so that the quotient in question is of exponent 2. Since $Z$ wr $B \in \mathcal{S}_{2}, Z_{2}$ wr $B \in \mathcal{S}_{2}$ and $\left(Z_{2}\right)^{n}$ wr $B$ and finally $\left(Z_{2}\right)^{n}$ wr $\left(B+Z_{2}\right) \in \mathcal{S}_{2}$. Therefore because $\left(\left(A_{i+1}+A_{i+1}^{g}\right) / C_{i}\right) \cdot\left(B+Z_{2}\right) \subseteq\left(Z_{2}\right)^{n}$ wr $\left(B+Z_{2}\right)$, there is an $\mathcal{S}_{2}$-refinement of $C_{i}<A_{i+1}+A_{i+1}^{g}$ by groups normalized by $B+Z_{2}$. Since $L$ is normal, this is sufficient.

(3) If $|B|=8$, then $Z$ wr $B \in \mathfrak{S}_{2}$ if, and only if, $B$ is Abelian of exponent 2 or 4 , or dibedral.

Proof. This merely excludes $Z_{8}$ and $Q$, the quaternions. $Z_{8}$ has been excluded above. In the group ring $R(Q)$ of the quaternions over the rationals, the subspace

$$
V=\{a \cdot 1+(-a) \cdot(-1)+b \cdot i+(-b) \cdot(-i)+c \cdot j+(-c)(-j)+d \cdot k+(-d)(-k)\}
$$

is four-dimensional and has no $Q$-invariant subspaces. Being $R$-irreducible makes it $Z$-irreducible. Therefore $Z$ wr $Q \notin \mathcal{S}_{2}$. The groups $B=Z_{2}+Z_{2}+Z_{2}, Z_{2}+Z_{4}$ and Dih (8) do satisfy $Z$ wr $B \in \mathcal{S}_{2}$. The first two follow from (2), while the third is in Curtis and Reiner [3, p. 505].

(4) $Z \operatorname{wr}(\operatorname{Sym} 3) \in \mathcal{S}_{2}[3$, p. 505].

(5) $Z$ wr $B \in \mathcal{S}_{2}$ where $B$ is dibedral over an elementary Abelian 3-group. 
Proof. Let $B=C \cdot Z_{2}$ where $|C|=3^{n}$. Over the complex numbers, $B$ has irreducible representations the squares of whose degrees sum to $2 \cdot 3^{n}$. There are two of degree one and one of degree two corresponding to each subgroup of $C$ of index 3 (taking the subgroup as a kernel). This accounts for $2+1 / 2\left(3^{n}-1\right)$ representations and, in the sum of squares formula, we have now accounted for $1+1+$ $1 / 2\left(3^{n}-1\right) \cdot 4=2 \cdot 3^{n}=|B|$, and therefore all the irreducibles. By (4), these are all realizable over $Z$.

(6) $Z$ wr $B \in \mathcal{S}_{2}$ where $B$ is dibedral over an Abelian group of exponent 4.

Proof. Use a counting argument similar to the above, and (3). We may assume $C$ is now of order $4^{n}$ for additional summands of order 2 are taken care of by (2). $B / B^{\prime}$ is an elementary Abelian 2-group of order $2^{n+1}$ so there are $2^{n+1}$ representations of degree one. The number of subgroups $D$ with $C / D$ cyclic of order 4 is $1 / 2\left(4^{n}-2^{n}\right)$ so we have accounted for $2^{n+1}+1 / 2\left(4^{n}-2^{n}\right) \cdot 4=1 / 2\left(4^{n+1}\right)=2 \cdot 4^{n}=$ $|B|$ and therefore all.

(7) If $B$ is finite Abelian, then $Z$ wr $B \in \mathcal{S}_{2}$ if and only if $\exp (B)=1,2,3$, 4,6 .

Proof. By (1), (2), (5) and (6).

The remaining results we have obtained are fragmentary and consist of special subdirect sums of Dih (8). (6) may be viewed as a positive case, while if $\operatorname{Dih}(8)=\left\langle a, b \mid a^{4}=b^{2}=1, b^{-1} a b=a^{-1}\right\rangle$ the subgroup $B=\langle(a, b),(b, a)\rangle \subset$ $\operatorname{Dih}(8)+\operatorname{Dih}(8)$ is of order 32 and $Z$ wr $B \notin \mathcal{S}_{2}$ (for $B /\left\langle\left(a^{2}, a^{2}\right)\right\rangle$ is the quaternions). We note also that $Z$ wr $\left(\operatorname{Dih}(8) \times Z_{3}\right) \notin \mathcal{S}_{2}$ since $Z_{5}$ wr $\left(\operatorname{Dih}(8) \times Z_{3}\right) \notin \mathcal{S}_{2}$ by Lemma 3 (iv); and $Z$ wr $\left(\operatorname{Dih}(8) \times Z_{4}\right) \notin \mathfrak{S}_{2}$ since $Z_{3}$ wr $\left(\operatorname{Dih}(8) \times Z_{4}\right) \notin \mathfrak{S}_{2}$.

\section{REFERENCES}

1. J. M. Bateman, On the solvability of unit groups of algebras, Trans. Amer. Math. Soc. 157 (1971), 73-86. MR $43 \# 2118$.

2. Roger Carter and Paul Fong, The Sylow 2-subgroups of the finite classical groups, J. Algebra 1 (1964), 139-151. MR 29 \#3548.

3. Charles W. Curtis and Irving Reiner, Representation theory of finite groups and associative algebras, Pure and Appl. Math., vol. 11, Interscience, New York, 1962.

MR $26 \# 2519$.

4. John R. Durbin, Finite supersolvable wreath products, Proc. Amer. Math. Soc. 17 (1966), 215-218. MR 32 \#4192.

5. K. W. Gruenberg, Residual properties of infinite soluble groups, Proc. London Math. Soc. (3) 7 (1957), 29-62. MR 19, 386.

6. B. Huppert, Endliche Gruppen. I, Die Grundlehren der math. Wissenschaften, Band 134, Springer-Verlag, Berlin, 1967. MR 37 \#302.

7. Hanna Neumann, Varieties of groups, Ergebnisse der Mathematik und ihrer Grenzgebiete, Band 37, Springer-Verlag, Berlin, 1967. MR 35 \#6734. 
8. Donald B. Parker, Wreath products and formations of groups, Proc. Amer. Math. Soc. 24 (1970), 404-408. MR 40 \#5739.

9. W. R. Scott and L. M. Sonneborn, Supersolvable wreath products, Math. Z. 92 (1966), 154-163. MR $33 \# 178$.

10. D. Suprenenko, Soluble and nilpotent linear groups, Ministerstvo Vyš̌. Obražovanija SSSR, Izdat. Belgosuniv. im. V. I. Lenina, Minsk, 1958; English transl., Transl. Math. Monographs, vol. 9, Amer. Math. Soc., Providence, R. I., 1963. MR 27 \#4861a, b.

DEPARTMENT OF MATHEMATICS, MICHIGAN STATE UNIVERSITY, EAST LANSING, MICHIGAN 48823 